\title{
EMPRENDIMIENTO, INNOVACIÓN Y EDUCACIÓN: TRILOGÍA ESENCIAL PARA EL DESARROLLO
}

\section{Entrepreneurship, Innovation and Education: Trilogy essential for development}

\author{
Lida K. Sandoval Guerrero ${ }^{1}$ \\ Universidad UTE \\ lida.sandoval@ute.edu.ec \\ Juan D. Martínez Guevara² \\ Instituto Superior Tecnológico Superior YAVIRAC \\ juandoenitz1956@gmail.com
}

\section{Ensayo Académico}

Fecha de recepción del artículo: 11/2018

Fecha de aceptación definitiva: 12/28/2018

1 Docente a tiempo completo en la Facultad de Comunicación, Artes y Humanidades de la Universidad Tecnológica Equinoccial (Quito, Ecuador). Magister en Administración y Dirección de Empresas por la Universidad Israel (Quito, Ecuador), doble titulación Universidad República Oriental del Uruguay. Candidata Phd en Educación por la Universidad Católica Andrés Bello (Caracas, Venezuela). Correo electrónico: lida.sandoval@ute.edu.ec.

2 Magister en Administración y Dirección de Empresas por la Universidad Israel (Quito, Ecuador). Docente tiempo completo del Instituto Superior Tecnológico Superior Yavirac (Quito, Ecuador). Correo electrónico: juandoenitz1956@gmail.com. 
Lida K. Sandoval Guerrero - Juan D. Martínez Guevara

\section{Emprendimiento, innovación y educación: trilogía esencial para el desarrollo}

La educación ha sido y será el medio idóneo para desarrollar y fortalecer las capacidades de los individuos.

Lida Sandoval Guerrero

\section{RESUMEN}

Emprender es una actividad innata de los seres humanos cuando existen dificultades a ser resueltas — sean estas de carácter intrínseco o extrínseco-; esta situación motiva la formación de organizaciones empresariales que dinamizan la economía de un país y que se han convertido en su eje de desarrollo. En tal razón, la presente investigación es de carácter cualitativo; se ha realizado mediante el análisis documental de tres aspectos (emprendimiento, innovación y educación) con la finalidad de identificar la incidencia de esta última como un factor preponderante en la formación o desarrollo de capacidades emprendedoras en los estudiantes y futuros emprendedores, y de la importancia de la relación entre emprender e innovar como medios esenciales para mejorar las posibilidades de permanencia de este tipo de negocio. Los resultados de este estudio revelaron que la capacidad de emprendimiento a escala nacional es superior que en la región, pero la carencia de conocimiento, posibilidades de inversión y mantenimiento de los emprendimientos conducen al fracaso y la pérdida de capacidades de generación de ingresos importantes. Esta realidad es conocida por las entidades involucradas, aquellas que en los últimos años han dado paso a iniciativas que promuevan el desarrollo de capacidades específicas, así como de medios de asesoría empresarial y financiamiento.

Palabras claves: emprendimiento, innovación, educación, cultura emprendedora.

\section{ABSTRACT}

Entrepreneurship is an innate human activity when there are difficulties to be solved - be they intrinsic or extrinsic; this situation motivates the formation of business organizations that stimulates a country's economy and that has become its axis of development. In this regard, the present investigation is qualitative in nature; it has been carried out through the documentary analysis of three aspects (entrepreneurship, innovation and education) in order to identify the incidence of the latter as a preponderant factor in the formation or development of entrepreneurial skills in students and future entrepreneurs; and the importance of the relationship between entrepreneurship and innovation as an essential mean to improve the possibilities of permanence of this type of business. The results of this study revealed that entrepreneurship capacity at the national level is higher than in the region, but the lack of knowledge, investment possibilities and maintenance of the ventures lead to the failure and loss of important income generation capacities. This reality is known by the entities involved, those that in recent years have given way to initiatives that promote the development of specific capacities, as well as means of business advice and financing.

Keywords: entrepreneurship, innovation, education, entrepreneurial culture. 
Lida K. Sandoval Guerrero - Juan D. Martínez Guevara

\section{Emprendimiento, innovación y educación: trilogía esencial para el desarrollo}

\section{Introducción}

Con el devenir del tiempo, el desarrollo académico, social y económico de los seres humanos se ha convertido en una necesidad constante, toda vez que la búsqueda del mejoramiento de su calidad de vida, desde diferentes perspectivas y paradigmas, es el hito fundamental de cada uno de ellos. En este escenario surge una interrogante: ¿de qué manera la educación amalgama la relación entre el emprender y el innovar? La respuesta más inmediata sería que educar es el pilar fundamental de una sociedad en desarrollo, por lo que las capacidades específicas de los emprendedores - crear e innovar - suelen en la mayoría de los casos estar ligadas al conocimiento, a las destrezas, a la actitud y aptitud del individuo.

Se considera que la educación formal, adquirida en la academia, o informal, en la cotidianeidad, determina que los niveles de competitividad laboral sean cada vez más depurados y perfeccionados; de allí que el desarrollo y la producción de nuevas ideas, productos y servicios se sustentan en un fuerte contenido científico, técnico y tecnológico. La educación ha sido y será el medio idóneo para desarrollar y fortalecer las capacidades de los individuos.

En la actualidad, la formación de capacidades se nutre del sustento educativo, pero para que tengan asidero en el alcance de metas e ideales individuales o comunitarios, se requiere que todos encontremos relaciones vinculantes para que el impacto de lo aprendido aporte al crecimiento socioeconómico, personal y profesional. Este es el punto de partida de nuestra propuesta investigativa, que intenta dejar en evidencia la necesidad de formar profesionales cualificados con alto nivel de conocimiento, cuyas habilidades sean fortalecidas mediante su experiencia laboral, reconociendo que para ser empresario, el primer paso es aprender a ser emprendedor.

A través de un proceso dialéctico, hemos encontrado la lógica de conceptualizar a la educación como el hito fundamental de una sociedad que busca progresar. Asimismo, reconocemos la importancia de que los docentes universitarios, formadores de seres humanos, somos los responsables de forjar mentes emprendedoras y creativas que entiendan a la capacidad de innovación como esencia del progreso.

Así, surge una trilogía cognitiva y experiencial que entrelaza lo que el estudiante conoce, lo que sabe hacer, y lo que le gusta hacer con pasión, cuyo impacto debe tener un fin: "el crear espacios de emprendimiento que generen no solo nuevas fuentes de ingresos, sino que formen una nueva sociedad sustentada en el paradigma 'ganarganar"' (Covey, 2015, p. 47). 
Lida K. Sandoval Guerrero - Juan D. Martínez Guevara

\section{Emprendimiento, innovación y educación: trilogía esencial para el desarrollo}

\section{Emprender: el inicio de un nuevo estilo de vida}

Uno de los términos que en el ámbito socioeconómico se menciona con relativa frecuencia — debido a su cercana relación con la dinámica productiva de un país - es el emprender. Está asociado a la acción de dar inicio a una actividad económica que busca satisfacer a un sinnúmero de necesidades de las personas que lo ponen en práctica y poseen características 'emprendedoras'. Esto impulsa al crecimiento económico y a la formación de un nuevo estilo de vida, a partir de un cambio paradigmático que fortalece la cultura empresarial, pilar fundamental para el desarrollo.

Desde este punto de vista, el término en cuestión se asocia con el arranque de un nuevo negocio. Alcaraz Rodríguez (2006, p. 21) sostiene que en "el ámbito de los negocios el emprendedor es un empresario, es el propietario de una empresa comercial con fines de lucro". Esta acepción coincidencia con la de Torrent-Sellens (2014, p. 39), quien define la función del emprendedor como la "creación de empresas, de manera que la persona o personas que desarrollan nueva actividad económica aportan este tipo de servicio, el trabajo emprendedor".

El término 'emprender' fue utilizado por primera vez por Schumpeter, ${ }^{3}$ en el año de 1934, con el fin de identificar a quien comenzaba una empresa. El término fue ligado a empresarios innovadores, refiriéndose a aquellos sujetos que con sus actividades aportan la economía de un país, debido a la dinámica comercial, mejorando niveles de ingresos, promoviendo plazas de trabajo y disminuyendo la crisis económica. Los emprendedores son vistos como agentes de cambio y de desarrollo económico.

Según la Real Academia Española (RAE, 2018, p. 1), el término emprender proviene del latín in (en) y prendĕre (coger), cuya traducción lo define como "acometer y comenzar una obra, un negocio, un empeño, especialmente si encierran dificultad o peligro". La ejercicio de emprender está asociado con otras acciones relacionadas a dar inicio a nuevas experiencias, entre ellas la académica, en que el "vocablo denota un perfil, un conjunto de características que hacen actuar a una persona de manera determinada y le permite mostrar ciertas competencias para visualizar, definir y alcanzar objetivos" (Alcaraz Rodríguez, 2006, p. 21). La asociación entre emprendimiento y formación académica se convierte en una necesidad imperiosa, pues el conocer sobre la línea de negocio que se decide emprender, reduce los niveles de riesgo al fracaso.

Joseph Alois Schumpeter fue un destacado economista austro-estadounidense, Ministro de Finanzas en Austria y profesor de la Universidad de Harvard desde 1932 hasta su muerte. 
Lida K. Sandoval Guerrero - Juan D. Martínez Guevara

\section{Emprendimiento, innovación y educación: trilogía esencial para el desarrollo}

Las definiciones reflexionadas permiten concluir que el emprendimiento es la actitud y aptitud de las personas que presentan capacidades para generar algo nuevo e innovador, y que, en la constante búsqueda de la solución de problemas, dan origen a nuevas fuentes de ingresos.

\section{Factores que limitan y promueven el emprendimiento}

Según datos proporcionados por la Global Entrepreneurship Monitor (GEM), ${ }^{4}$ los resultados de la encuesta nacional de expertos, en el 2018 se han destacado los siguientes factores que fomentan o restringen al emprendimiento, ya sea por su grado de avance, consolidación o importancia: educación y capacitación, infraestructura comercial y profesional, acceso a infraestructura física, información y transferencia de I\&D. Con menor predominio mencionan: programas gubernamentales y fuerza laboral, clima económico, normas sociales y culturales, y apertura de mercados. Entre estos últimos aspectos, la fuerza laboral fomenta el emprendimiento no por su buen estado, sino porque el desempleo impulsa el emprendimiento por necesidad. En cuanto a la apertura de mercado, se destacan las oportunidades del Acuerdo Comercial con la Unión Europea (GEM, 2018).

\section{Competencias emprendedoras: requerimiento del emprendedor actual}

Tomando en consideración que todas las personas son capaces de realizar actividades en diferentes niveles de competitividad, el ser emprendedor ha generado polémica tanto en su acepción como en su aplicabilidad. Se se lo asocia con la pregunta: ¿el emprendedor nace o se hace? Este cuestionamiento invita a repensar en las características que permiten diferenciarlo de una persona común.

Partiendo de la definición de competencia laboral dada por Martha Alles (2005), estas características se denotan a través de la personalidad de un individuo, evidenciadas por el comportamiento y la tendencia de la búsqueda del éxito en sus actividades productivas, que en virtud de las competencias emprendedoras, se relacionan a la puesta en marcha, manejo y productividad de un negocio exitoso. En tal

GEM: Global Entrepreneurship Monitor, iniciado el año 1998 por el Babson College y la London Business School. Es un proyecto de investigación que surgió, precisamente, con la intención de analizar la actividad emprendedora, así como los factores que influyen en la creación de nuevas empresas. Este proyecto analiza de manera exhaustiva la relación que hay entre la actividad emprendedora y el crecimiento económico de los países. 
Lida K. Sandoval Guerrero - Juan D. Martínez Guevara

\section{Emprendimiento, innovación y educación: trilogía esencial para el desarrollo}

razón, el desempeño de sus actividades se sustenta en el conocimiento formal adquirido en el claustro académico, y el conocimiento informal o empírico sustentado por la experiencia y la noción del saber hacer.

Otro aporte importante es el de McClelland (1973, p. 4), quien define al ser competitivo como una "característica subyacente de una persona que le permite demostrar un desempeño superior en un determinado puesto, rol o situación diferenciado entre personas con desempeño excelente versus personas con desempeño promedio".

Desde la perspectiva de la economía, Fernández y Ruiz (2006, p. 32) establecen las competencias del emprendedor, al mencionar que la diferencia entre el empresario capitalista y el emprendedor: "el capitalista es meramente el poseedor del negocio y, por tanto, no es un emprendedor en sí, sino alguien que participa en cierto riesgo y obtiene beneficio de ello".

Estos sustentos teóricos permiten determinar que los emprendedores son personas que poseen características muy específicas en su campo de acción, y se diferencian de un empresario, en su capacidad de innovación. Poseen la habilidad de generar algo nuevo, con valor agregado, a partir de la combinación de recursos; su capacidad de superar el temor al riesgo de pérdida en una inversión —debido a su exactitud en el análisis económico y manejo adecuado de recursos- les permite minimizar el fracaso.

\section{Innovación: origen del término y su definición}

Proviene etimológicamente de la voz latina innovare, cuyo significado es "cambiar o alterar las cosas incorporando novedades" (Medina Salgado y Espinosa Espíndola, 1994, p. 6). Esta definición la comparte la Real Academia de la Lengua (1986, p. 462) en el que se define al término como "mudar o alterar las cosas introduciendo novedades".

En el sentido estricto, el término innovación se relaciona con la generación de aspectos nuevos o diferentes, relacionados con la mejora de productos o bienes, servicios o procedimientos ya creados. En este sentido, se diferencia de la invención, la cual engloba nuevas ideas que generan prototipos o nuevos conceptos. La innovación se relaciona con la puesta en valor, es decir con la generación de valores agregados o ventajas competitivas a productos (bienes) o servicios que están asociados al mejoramiento de la calidad de vida de la sociedad. Estos apuntes se relacionan con la necesidad de generar soluciones a los problemas identificados, muchos de ellos 
Lida K. Sandoval Guerrero - Juan D. Martínez Guevara

\section{Emprendimiento, innovación y educación: trilogía esencial para el desarrollo}

congruentes con el mejoramiento de la calidad de vida de las personas. Algunos autores relacionan la innovación con términos como ventaja competitiva, valor agregado o aspecto diferenciador, vinculados con lo novedoso, lo nuevo o lo diferente.

Para clarificar la definición dada desde el punto de vista epistemológico, han surgido múltiples acepciones en torno al término innovación, que se relacionan en tres ejes de acción: novedoso, transformacional y beneficioso. El primero está relacionado con la dimensión de singularidad, algo nuevo, diferente, actual, desconocido hasta el momento (contextual-objetiva). El segundo se vincula con la dimensión de cambio, relacionado con la temporalidad, pues determina modificaciones en productos (bienes) o servicios que son modificados en relación de las necesidades de los requirentes. Finalmente, el tercero hace referencia a la dimensión de beneficioso o ventajoso para la comunidad, lo cual determina la evolución y el progreso social.

Dentro de las múltiples definiciones del término innovación es importante considerar el pensamiento económico de Schumpeter (1978), quien considera a la innovación como un fenómeno empresarial. De esta forma se relaciona con la incidencia de factores económicos, sociales y culturales en el impulso para el desenvolvimiento ${ }^{5}$ del sistema económico de un país, por lo que la conceptualizó como la generación de nuevos métodos y procedimientos que promueven la introducción de bienes y servicios mejorados en el mercado.

Para este autor, la innovación es la capacidad e iniciativa que una persona involucrada en el mundo empresarial es capaz de generar nuevas combinaciones en los medios de producción. Por ende, la dinámica económica de un país está relacionada con la competencia innovadora, de allí que la reciprocidad de la innovación con la educación y el emprendimiento es directa.

El término innovación es confundido como invención de algo nuevo o diferente; respecto a ello Freeman (1975), citado por Pere Escoras Castells y Jaume Valls Pasola (2003, p. 25), menciona que su concepto es mucho más amplio que el de invención, pues este último consiste en crear cosas nuevas, es "una idea, un boceto o un modelo para un dispositivo, producto o sistema nuevo o perfeccionado", mientras que innovar implica poner en marcha lo generado. No termina hasta "la puesta al mercado de la invención", lo que corrobora que el punto de vista de Freeman y Schumpeter comprende

La obra original de Schumpeter fue publicada en alemán en el año de 1912, con el título Theorie der wirstschanftlichen Entwicklung. Según nota de su traductor a la versión epañola (J. Prados Arrarte), el mismo schumpeter ha preferido que se utilice la palabra desenvolvimiento a la de evolución, tpérmino con el cual se dio a conocer su obra, debido al título utilizado en la versión al inglés The Theory of Economic Development. 
Lida K. Sandoval Guerrero - Juan D. Martínez Guevara

\section{Emprendimiento, innovación y educación: trilogía esencial para el desarrollo}

a la innovación como el hecho empresarial que dinamiza económica de un país, a partir de la capacidad de emprendimiento de los grupos sociales que no es propia de inversores o inventores.

Se puede concluir entonces que innovar es generar cosas nuevas y diferentes que mejoren notoriamente los prototipos o aspectos creados, ante la necesidad de crear cosas nuevas, que inviten a solucionar problemas identificados. La capacidad de innovar se encuentra relacionada no solo con las aptitudes de las personas, sino con la educación, sea esta de carácter formal (conocimientos adquiridos de forma regulada, en un proceso de educación integral o regulado) o informal (conocimientos adquiridos de manera libre y experiencial).

El fomento de la innovación necesita el diálogo entre los investigadores de la educación y los prácticos; deben ser involucrados también las personas que toman decisiones y los grupos de la sociedad civil que se interesan en la educación. La innovación depende de la capacidad de los actores para establecer nuevas relaciones entre la educación y la realidad exterior a ella. Todas las experiencias de innovación tienen como prioridad la formación permanente de involucrados en los procesos de emprendimiento y la producción de bienes y servicios.

\section{Educación: eje de desarrollo del emprendimiento y la innovación}

Los procesos de globalización, al igual que los avances tecnológicos, la innovación, los sistemas de mercado cada día más crecientes por el nacimiento de nuevas formas de negocios (emprendimientos) y las demandas del mercado laboral por captar personas con más capacidades para un mejor desempeño, han hecho que los Estados busquen continuamente mejoras en los sistemas educativos, con el fin de dar una respuesta efectiva a los requerimientos de la economía de cada país. En este proceso surgen diversas ofertas educativas, cuyos componentes curriculares han incorporado asignaturas relacionadas con el desarrollo de capacidades emprendedoras, que mediante su aplicabilidad en el ejercicio de enseñanza-aprendizaje, buscan el desarrollo de capacidades de los educandos, desde la triangulación e integración de tres ejes: conocimientos, destrezas y valores y principios, con la finalidad de que el estudiante ponga en la práctica los conocimientos adquiridos, integrando el saber, el saber hacer, el saber ser y el saber convivir (Delors, 1994).

Se puede establecer que la educación es una orientación que procura dar contestación a los cambios sociales, culturales, económicos, ecológicos y los avances 
Lida K. Sandoval Guerrero - Juan D. Martínez Guevara

\section{Emprendimiento, innovación y educación: trilogía esencial para el desarrollo}

tecnológicos, mediante la formación de personas competentes con capacidades que den respuesta ante la problemática en cualquier contexto. Para la formación de estudiantes hacia el desempeño adecuado en diversos ámbitos se requiere que sean los protagonistas de su propia formación y el docente se convierta en un mediador de conocimientos, para el desarrollo y fortalecimiento de las habilidades cognitiva y metacognitivas. ${ }^{6}$

El clima emprendedor en el Ecuador ha mejorado notoriamente en los últimos años debido al aporte e interés de las autoridades del sistema educativo. En el bachillerato, el Ministerio de Educación (MinEduc) ha incluido en el currículo la asignatura de Emprendimiento y Gestión, cuyo origen proviene del contexto legal y constitucional, dado en la Constitución de 2008 (arts. 283, 284, 302, 304 y 306), el Plan de Desarrollo Nacional y la Ley de Economía Popular y Solidaria. El objetivo de esta incorporación es que el "estudiante haya desarrollado sus capacidades emprendedoras y que, con la aplicación diaria de estas capacidades, se convierta en una persona dinamizadora de la sociedad en su conjunto, de su familia, zona geográfica o ciudad y, pueda generar fuentes de trabajo" (Ministerio de Educación, 2015, p. 7).

Asimismo, en la educación superior se ha incorporado en el currículo de todas las carreras la asignatura de emprendedores, con el fin de desarrollar las capacidades específicas en esta área, fomentar e impulsar el deseo de emprender, con un fuerte componente científico-técnico, en el cual se evidencia tres factores de interrelación activa que permiten dinamizar la economía nacional, reduciendo el porcentaje de fracasos.

- Socioeconómico: permite desarrollar posibilidades de generar emprendimientos que dinamicen la economía nacional, a través de la puesta en marcha de las capacidades emprendedoras de los estudiantes universitarios del país.

- Empresarial: vincula la Universidad y el ámbito laboral, promoviendo el acercamiento entre academia-sociedad.

- Curricular: permite interrelacionar las necesidades de formación integral de los individuos mediante la implementación de un currículo abierto al cambio, flexible que promueva la integración de factores del contexto.

Metacognición: a primera vista se trataría de definirla como la cognición sobre la cognición, el conocimiento sobre el conocimiento, el pensamiento sobre el pensamiento (Mayor y GonzálezMárquez, 1993). 


\section{Emprendimiento, innovación y educación: trilogía esencial para el desarrollo}

Figura. 1. Factores de interrelación para el desarrollo de capacidades emprendedoras

\section{SOCIOECONÓMICO}

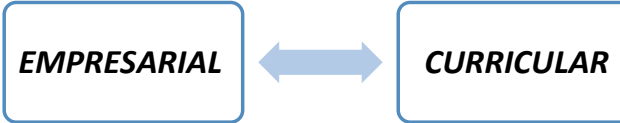

Fuente: Lida K. Sandoval Guerrero

La formación académica que potencialice el desarrollo de capacidades emprendedoras en los estudiantes, se ha convertido en el Ecuador en una estrategia. En el ámbito educativo surge la necesidad de reconocer la importancia de incorporar como resultado de aprendizaje en todas las carreras, las capacidades emprendedoras, alineadas desde la estrategia hasta la conformación de la malla curricular.

\section{Discusión}

El equipo Global Entrepreneurship Monitor (GEM) reveló que el Ecuador mantiene el índice de Actividad Emprendedora Temprana (TEA ${ }^{7}$ más alto entre los países de América Latina y el Caribe; como resultado de las iniciativas emprendedoras en el país se obtuvo un indicador de 29,6\%. Esta información determina que el Ecuador es uno de los países con mayor actividad en generación de emprendimientos en toda la región. $\mathrm{A}$ pesar de ello, no hay una ley que regule e impulse al sector emprendedor. Según datos publicados por la prensa nacional, de acuerdo al grupo GEM, en el país "tres de cada diez adultos emprenden en el Ecuador" (GEM, 2018).

Nuestro país es uno de los más emprendedores, ocupa "el tercer lugar a nivel mundial con un TEA del $29,6 \%$ superior a la media mundial que es de $9,40 \%$, lo que significa que el $27,24 \%$ de los ecuatorianos están involucrados en una actividad emprendedora" (GEM, 2018). En la siguiente figura se puede apreciar la evolución del TEA en el Ecuador.

TEA es una medida de la actividad emprendedora individual y como tal responde a las decisiones de emprender. Estas están influenciadas por las condiciones del entorno, discutidas en el capítulo anterior, por las actitudes de la sociedad hacia el emprendimiento y las percepciones sobre este, que componen la cultura social que soporta en diverso grado a los emprendedores. 
Lida K. Sandoval Guerrero - Juan D. Martínez Guevara

Emprendimiento, innovación y educación: trilogía esencial para el desarrollo

Figura. 2. Evolución del TEA en el Ecuador

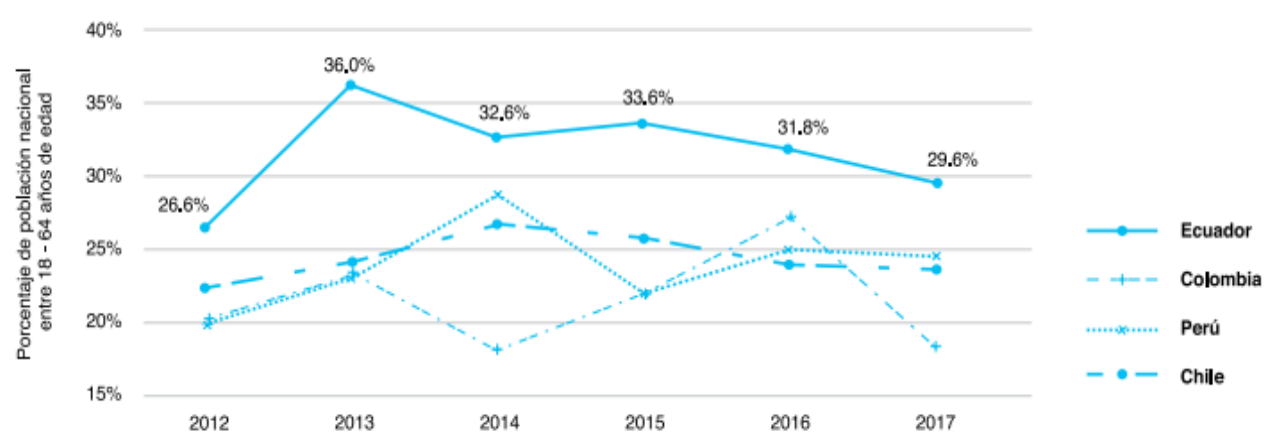

Fuente: Espae, Espol y GEM Ecuador

El Ecuador es considerado entre los países de la región con mayor índice de emprendimiento, de acuerdo a la información revelada en el informe GEM.

Figura. 3. Países de TEA más alta en la región

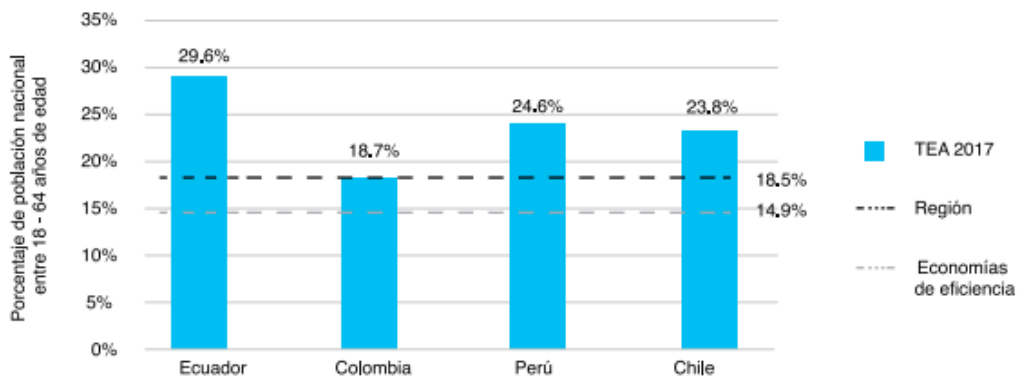

Fuente: Espae, Espol y GEM Ecuador

Los datos mencionados fueron emitidos por Virginia Lasio, directora del GEM Ecuador, al detallar que:

[...] alrededor de 3 millones de adultos, de entre 18 y 64 años, empezaron el proceso de puesta en marcha de un negocio (emprendimiento naciente) o poseían uno con menos de 42 meses de antigüedad (emprendimiento nuevo). Esto, según Lasio, representa el $29,6 \%$ de la población adulta, siendo la TEA más alta de la región, seguido por Perú con el $24,6 \%$ y Chile con el $23,8 \%$. La medición recogió información de 2.060 personas a nivel nacional y de 37 expertos. (GEM, 2018, p. 7)

Sin embargo, la mayoría de los emprendimientos son de autoempleo, no son dinámicos; la mayoría de ellos no llegan a subsistir al tercer año de vida, lo que revela que sus organizaciones no son estables, debido al desconocimiento de niveles de gestión de los propietarios. 


\title{
Emprendimiento, innovación y educación: trilogía esencial para el desarrollo
}

\begin{abstract}
En Ecuador el 42,3\% de la población emprenden por necesidad, por falta de ingresos; mientras que el $36,7 \%$ lo hacen por oportunidad de mejoras. En los demás países de la región, como Chile (59,7\%), Perú (62,3\%), Colombia (59,4\%), se emprende mayormente por oportunidad de mejoras. Otra variable del estudio es el emprendimiento sénior. La tea sénior en 2017 se ubicó en el $25,30 \%$, la más baja de los últimos cuatro años. Sin embargo, los séniores (de 50 a 64 años) emprenden casi a la par que los jóvenes (de 18 a 29 años) en Ecuador. (GEM, 2018, p. 7)
\end{abstract}

De acuerdo a los estudios realizados por la Espea de la Escuela Politécnica del Litoral (Espol), en el Ecuador demográficamente se presentan las siguientes características: el 52,85\% de los emprendedores tienen menos de 35 años, el 68,1\% tienen como nivel de educación primaria o secundaria y finalmente su área de ubicación es del $48,8 \%$ en hogares con un ingreso mensual desde del salario básico hasta los $\$$ 750,00 USD; esto determina que la mayor parte de emprendimientos surgen por necesidades económicas, como se aprecia en la siguiente figura (GEM, 2018).

Figura. 4. Demografía de los emprendedores

Demografía de los emprendedores

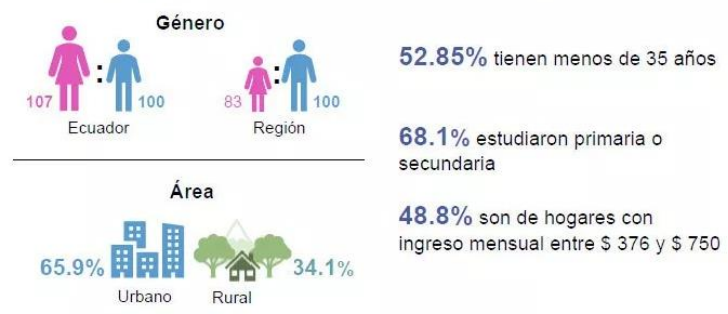

Fuente: Espae, Espol, GEM Ecuador

Es importante señalar que en el país existe un organismo rector en el área de productividad que tiene cobertura en el ámbito del emprendimiento a través de la Dirección de Desarrollo de MIPYMES y Emprendimiento, de la Subsecretaría de Desarrollo de MIPYMES y Artesanías del Ministerio de Industrias y Productividad del Ministerio de Industrias y Productividad (Mipro, 2014), cuya finalidad es:

Impulsar el desarrollo del sector productivo industrial y artesanal, a través de la formulación y ejecución de políticas públicas, planes, programas y proyectos especializados, que incentiven la inversión e innovación tecnológica para promover la producción de bienes y servicios con alto valor agregado y de calidad, en armonía con el medio ambiente, que genere empleo digno y permita su inserción en el mercado interno y externo. 
Lida K. Sandoval Guerrero - Juan D. Martínez Guevara

\section{Emprendimiento, innovación y educación: trilogía esencial para el desarrollo}

Para cumplir con esta declaración, dicha entidad ha implementado, entre varios otros, el proyecto denominado Centros de Desarrollo Empresarial (CDE) (Mipro, 2014). Según datos del Mipro, en la actualidad se cuenta con 89 CDE; esto se ha realizado en un esfuerzo conjunto de los Gobiernos Autónomos Descentralizados (GAD) y universidades que buscan impulsar el emprendimiento en el país.

Las dificultades que enfrentan los emprendedores son: la falta de proveedores de servicios de desarrollo empresarial, limitado acceso al financiamiento (crédito o inversión), escasas carreras de emprendimiento, baja resistencia a la posibilidad de fracaso, bajo nivel asociativo, escasa innovación en los productos y servicios ofertados, entre otros.

Desde el sector privado también existe un enorme trabajo en favor del desarrollo del emprendimiento entre universidades y fundaciones como la YABT (Young American Business Trust, adscrita a la OEA), la Universidad del Pacífico, Kruger Labs y CONQUITO, por nombrar a algunas.

\section{Conclusiones}

El presente análisis ha permito determinar tres ejes de estudio: emprendimiento, innovación y educación, como actores esenciales para la dinámica económica del país. Mediante esta reflexión se ha logrado identificar que existen varias ponencias relacionadas a la temática de emprendimiento, término muy utilizado actualmente en el ámbito educativo y empresarial.

Una de las definiciones genéricas fue dada por la RAE, en que se establece al emprendimiento como la acción de llevar adelante una actividad; cuando se hace referencia a un negocio, se habla específicamente de un emprendimiento empresarial. El emprendedor es la persona que con tenacidad, esfuerzo y creatividad genera nuevas propuestas. Se pudo identificar que las características de los emprendedores son sus altos niveles de creatividad, innovación y persistencia.

Según el estudio realizado, fomentar la cultura emprendedora es fundamental en el país, pues a través de ella se genera articulación, asociatividad y capacitación con el sector empresarial.

El Ecuador ha evidenciado un nivel alto de emprendimiento, llegando a ocupar uno de los primeros lugares entre los países de la región. El desafío es que estas iniciativas pasen de ser una motivación por necesidad a una por oportunidad y se logre 
Lida K. Sandoval Guerrero - Juan D. Martínez Guevara

\section{Emprendimiento, innovación y educación: trilogía esencial para el desarrollo}

su permanencia, lo cual no se ha podido superar, evidenciándose el constante cierre y fracaso de emprendimientos jóvenes.

Asimismo, se ha podido evidenciar la carencia de un centro de investigación acerca de la tarea emprendedora, tanto desde el punto vista público como privado. Toda la información que actualmente se genera es una simple aproximación, por lo que es imprescindible la creación de un espacio en el cual se investigue, procese y se brinde capacitación y soluciones para mantener una cultura emprendedora activa.

Se pudo determinar que la Dirección de Desarrollo de Mipymes y Emprendimiento de la Subsecretaría de Desarrollo de Mipymes y Artesanías del Ministerio de Industrias y Productividad cuenta con el programa Centro de Desarrollo Empresarial (CDE), conformado por 89 centros distribuidos por todo el país, en coordinación con los Gobiernos Autónomos Descentralizados Municipales y Provinciales y con algunas universidades estatales. Los directivos de estas instituciones van a generar una fuerte sinergia para motivar el emprendimiento en el país.

Se han dado una serie de programas de varias instituciones públicas, como por ejemplo, el Fondo Nacional de Garantías de la Corporación Financiera Nacional. Sin embargo, hace falta promover un mayor impulso a la iniciativa privada por parte del Estado; crear un verdadero programa de financiamiento para los emprendedores que contemple la entrega de capital de riesgo, el cual no existe. Por el contrario, es el sector privado quien ha invertido efectivamente en el sector emprendedor, mediante la creación de redes de inversionistas ángeles como son Start-Up Ventures o Carana.

La mayoría de los emprendimientos, según el GEM, no nacen fruto de aprovechar una oportunidad de negocio, sino de la necesidad de subsistencia, razón por la cual no superen el tercer año.

\section{Referencias bibliográficas}

Alcaraz Rodríguez, R. (2006). El emprendedor de éxito (4ºdición ed.). México D. F.: McGraw Hill / Interamericana Editores, S.A. de C.V.

Alles, M. (2005). Gestión por Competencias el Diccionario (2da. edición ed.). Buenos Aires, México, Santiago y Montevideo: Granica.

Covey, S. R. (2015). Los siete hábitos de la gente altamente efectiva. Miami: Franklin Covey.

Delors. J. (1994). La educación encierra un tesoro: Informe de la Unesco de la Comisión Internacional sobre Educación para el siglo XXI. París: Santillana / Unesco. 


\section{Emprendimiento, innovación y educación: trilogía esencial para el desarrollo}

Escoras y Valls. (2003). Tecnología e innovación en la empresa. Barcelona: Universidad Politécnica de Catalunya.

Fernández Esquinas Manuel y Jorge Ruiz. (2006). Los jóvenes y la creación de empresas. Madrid: Consejo Superior de Investigaciones Científicas.

Freeman, C. (1975). Teoría económica de la innovación industrial. Barcelona: Alianza.

GEM. (2018, 08 08). Global Entrepreneurship Monitor. Espea / Espol. Obtenido de: http://espae.espol.edu.ec/wpcontent/uploads/documentos/GemEcuador2017.pdf.

González Arnao, V. (1986). Diccionario de la Real Académia de la Lengua. París: Vicente Salva .

Inciarte y Canquiz. (2008). Formación integral desde el enfoque por competencias (1era edición ed.). Maracaibo: Universidad de Zulia.

McClelland. D. C. (1973). Testing for competence rather than intelligence. Obtenido el 05 03, 2016, de: American Psychologist: http://www.apa.org/journals/amp.html.

Medina Salgado y Espinosa Espíndola. (1994). La innovación en las organizaciones modernas. Obtenido el 06 29, 2018, de: http://www.azc.uam.mx/publicaciones/gestion/num5/doc06.htm.

MinEduc. (2015). Curriculo del Bachillerato General Unificado: Emprendimiento y Gestión. Ministerio de Educación del Ecuador.

Mipro. (2014). Ministerio de Industrias y Productividad. Obtenido el 07 03, 2014, de: http://www.industrias.gob.ec/.

RAE. (2018, 10 05). En clave RAE, DLE. Obtenido de: http://dle.rae.es/srv/fetch?id=Esip2Nv.

Schumpeter, J. (1978). Teoría del desenvolvimiento económico. México D. F.: Fondo de Cultura Económica.

Torrent-Sellens, J. (2014). Emprendimiento innovador y microempresas en red: Diez ideas para salir de la crisis. Barcelona: UOC - Oberta OUC Publishing.

Villafranco, G. (2018, 01, 15). 6 factores para potenciar el emprendimiento en Latinoamérica . World Economic Forum. https://www.weforum.org. 
Lida K. Sandoval Guerrero - Juan D. Martínez Guevara

Emprendimiento, innovación y educación: trilogía esencial para el desarrollo

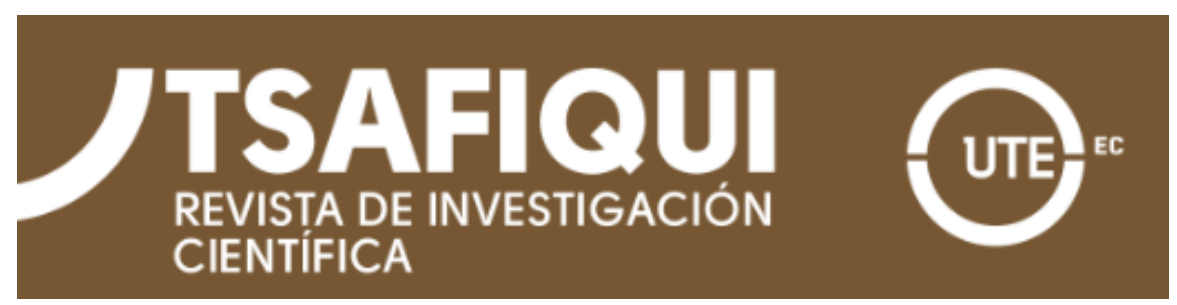

